\title{
Investigation of 3D Polynomial Model for the Geometric Correction of GeoEye Imagery
}

\author{
Samah Ramadan Mohamed Abo Ramadan ${ }^{1}$, Hafez Abbas Afify ${ }^{1}$, Sobhy Younes Abdelmonam ${ }^{1}$ \\ ${ }^{1}$ Department of Public Work Engineering, Faculty of Engineering, \\ Tanta University, Egypt \\ E-mail: samah.ramada@yahoo.com, hafezafify@yahoo.com, sobbhi100@yahoo.com
}

\begin{abstract}
GeoEye satellite has provided the world with high spatial resolution 0.50 meter panchromatic images. Not only the high spatial resolution, but also the high spectral, radiometric and temporal resolutions of GeoEye imagery make it ideally suited for mapping applications. Currently, the empirical sensor models are traditionally employed, instead of the physical models, to establish the mathematical relationships between the image space and the ground space. In this study, the geometric accuracy of pan GeoEye images was determined using three dimensional (3D) polynomial model. The obtained planimetric accuracy was compared to that obtained using the rational function model (RFM). The implementation of the 3D polynomial model (PM) experiments was performed using only the new standalone software EMAN, since the 3D PMs are not available in other software packages such as ERDAS imagine or PCI from Geomatica. However, both EMAN and PCI software were used to carry out the experiments for the RFMs. A GeoEye panchromatic image covering the city of Tanta, El Gharbiya, Egypt was used in this study. The results revealed that the $3 \mathrm{D}$ polynomial models have the capability for geometrically correcting GeoEye images since it provides competitive geometric accuracies, compared to those obtained using 3D RFMs.
\end{abstract}

Index Terms-High-resolution imagery, Geometric accuracy, GeoEye panchromatic images, Rational function model, Polynomial model, Orthoimages

\section{INTRODUCTION}

The demand for accurate and up to date spatial information is increasing and its availability is becoming more important for a variety of tasks. Today's commercial high-resolution satellite imagery offers the potential to extract useful and accurate spatial information for a wide variety of mapping and GIS applications [1].

Since its launch in April 2008, GeoEye satellite has been consistently providing high-resolution satellite images with 0.5 -meter in panchromatic mode. The availability of such high-resolution data from GeoEye mages have opened a new era heralding a promising future for mapping applications [2]. The orbit and sensor information during the scene acquisition time is not provided with GeoEye images and thus potentially precludes the application of the physical reality model in the geo-correction process. Instead, the empirical sensor models are used to describe the objectimage geometry. Several investigations have been already carried out to investigate geometric correction of GeoEye imagery using RFMs [3, 4].

EMAN software is a standalone software package that has been developed in MATLAB environment to allow the implementation of thirty-six empirical models for rectifying satellite images. These 36 empirical models are categorized by model direction (forward models and inverse models), model type (rational function models, rational function models with equal denominators and polynomial models), model order (third order model, second order model and first order model), and model dimension (three dimensions and two dimensions). EMAN software includes many tools to perform the different steps of rectification and orthoimage generation processes starting from collecting control points from the uncorrected image and the reference source, followed by selecting the empirical model and determining the unknown coefficients of that model then evaluating the computed model using check points and finally, generating the rectified images or the orthoimages.

This paper is devoted to investigate the performance of the 3D polynomial model for geometric correction of GeoEye imagery using only EMAN software package because the 3D PMs are not available in the most commonly software packages as ERDAS imagine and PCI from Geomatica Canada. A GeoEye panchromatic image covering the city of Tanta, El Gharbiya, Egypt was used. The geometric accuracies obtained using 3D PMs was compared to their counterparts obtained using RFMs. However, EMAN and PCI softwares were used to carry out the experiments of RFMs.

\section{MATHEMATICAL MODEL}

The rational function model (RFM) is one of the most commonly used empirical models in remote sensing applications. RFM is a pure mathematical model, which relates ground point coordinates to image pixel coordinates in the form of rational functions that are ratios of polynomials. Equations (1) to (4) represent the forward transformation from ground coordinates to image coordinates. RFM can also perform the inverse transformation from image coordinates to ground coordinates as shown in equations (5) to (8) [5]. 
$p=\frac{F_{1}(X, Y, Z)}{F_{2}(X, Y, Z)}$

$p=\frac{\sum_{i=0}^{m_{1}} \sum_{j=0 k=0}^{m_{2}} \sum_{j}^{m_{3}} a_{i j k} X^{i} Y^{j} Z^{k}}{\sum_{i=0}^{n_{1}} \sum_{j=0 k=0}^{n_{2}} \sum_{3} c_{i j k} X^{i} Y^{j} Z^{k}}$

$l=\frac{F_{3}(X, Y, Z)}{F_{4}(X, Y, Z)}$

$l=\frac{\sum_{i=0}^{m_{1}} \sum_{j=0 k=0}^{m_{2}} \sum_{3}^{m_{3}} b_{i j k} X^{i} Y^{j} Z^{k}}{\sum_{i=0}^{n_{1}} \sum_{j=0 k=0}^{n_{2}} \sum_{3} c_{i j k} X^{i} Y^{j} Z^{k}}$

$X=\frac{F_{5}(p, l, Z)}{F_{6}(p, l, Z)}$

$$
X=\frac{\sum_{i=0}^{m_{1}} \sum_{j=0 k=0}^{m_{2}} \sum_{3}^{m_{3}} e_{i j k} p^{i} l^{j} Z^{k}}{\sum_{i=0}^{n_{1}} \sum_{j=0 k=0}^{n_{2}} \sum_{3}^{n_{3}} g_{i j k} p^{i} l^{j} Z^{k}}
$$

$$
Y=\frac{F_{7}(p, l, Z)}{F_{8}(p, l, Z)}
$$

$$
Y=\frac{\sum_{i=0}^{m_{1}} \sum_{j=0 k=0}^{m_{2}} \sum_{3}^{m_{3}} f_{i j k} p^{i} l^{j} Z^{k}}{\sum_{i=0}^{n_{1}} \sum_{j=0 k=0}^{n_{2}} \sum_{3}^{n_{3}} h_{i j k} p^{i} l^{j} Z^{k}}
$$

Where,

$$
(p, l)
$$

$=$ The image coordinates.

$(X, Y, Z)$

$=$ The ground coordinates .

$a_{i j k}, b_{i j k}, c_{i j k}, d_{i j k}=$ The forward polynomial coefficients.

$e_{i j k}, f_{i j k}, g_{i j k}, h_{i j k}=$ The inverse polynomial coefficients.

In order to improve the numerical stability of the RFM equations and minimize the computational errors, all the image and ground coordinates are normalized to the range $[-1,1]$ by offsetting and scaling. For normalizing coordinates, the maximum and minimum of the coordinate values among fitting points are found. Scale and offset coordinates should be calculated in such a way that maximum value should be to $(+1)$ and minimum value to $(-1)$ [6]. The maximum power of each ground coordinate is typically limited to 3 ; and the total power of all ground coordinates is also limited to 3 .

For the third order inverse RFM the numerators and denominators are 20 term polynomial which have the form [7]:

$$
\begin{aligned}
& F(p, l, Z)=\sum_{i=0}^{m_{1}} \sum_{j=0}^{m_{2}} \sum_{k=0}^{m_{3}} e_{i j k} p^{i} l^{j} Z^{k} \\
& =e_{0}+e_{1} p+e_{2} l+e_{3} Z+e_{4} p l+e_{5} p Z+ \\
& e_{6} l Z+e_{7} p^{2}+e_{8} l^{2}+e_{9} Z^{2}+e_{10} p l Z+ \\
& e_{11} p^{2} l+e_{12} p^{2} Z+e_{13} l^{2} p+e_{14} l^{2} Z+ \\
& e_{15} Z^{2} p+e_{16} Z^{2} l+e_{17} p^{3}+e_{18} l^{3}+e_{19} Z^{3}
\end{aligned}
$$

Replacing equation (9) in equations (6) and (8) and eliminating the first coefficient in the denominator polynomial and putting the constant 1 instead, the third order inverse RFM form becomes:

$$
\begin{aligned}
& X=\frac{\left(1 p l Z \ldots l^{3} Z^{3}\right) \cdot\left(e_{0} e_{1} e_{2} e_{3} \ldots e_{18} e_{19}\right)^{T}}{\left(1 p l Z \ldots l^{3} Z^{3}\right) \cdot\left(1 g_{1} g_{2} g_{3} \ldots g_{18} g_{19}\right)^{T}} \\
& Y=\frac{\left(1 p l Z \ldots l^{3} Z^{3}\right) \cdot\left(f_{0} f_{1} f_{2} f_{3} \ldots f_{18} f_{19}\right)^{T}}{\left(1 p l Z \ldots l^{3} Z^{3}\right) \cdot\left(1 h_{1} h_{2} h_{3} \ldots h_{18} h_{19}\right)^{T}}
\end{aligned}
$$

There are 78 unknown coefficients in the third order inverse RFM. In other words, there are 39 unknown coefficients in each equation of the third order inverse RFM. In order to solve the third order RFM, at least 39 ground control points (GCPs) are required. The second order inverse RFM has 38 rational function coefficients (RFCs) and 19 RFCs in each equation as in equations (12) and (13). In such a case, 19 GCPs at least are required to determine the RFCs [8].

$$
\begin{aligned}
& X=\frac{\left(1 p l l Z \ldots l^{2} Z^{2}\right) \cdot\left(e_{0} e_{1} e_{2} e_{3} \ldots e_{8} e_{9}\right)^{T}}{\left(1 p l l z \ldots l^{2} Z^{2}\right) \cdot\left(1 g_{1} g_{2} g_{3} \ldots g_{8} g_{9}\right)^{T}} \\
& Y=\frac{\left(1 p l l Z \ldots l^{2} Z^{2}\right) \cdot\left(f_{0} f_{1} f_{2} f_{3} \ldots f_{8} f_{9}\right)^{T}}{\left(\begin{array}{llll}
1 & l & l & Z
\end{array} l^{2} Z^{2}\right) \cdot\left(1 h_{1} h_{2} h_{3} \ldots h_{8} h_{9}\right)^{T}}
\end{aligned}
$$

The first order inverse RFM has 14 rational function coefficients and 7 RFCs in each equation as shown in equations (14) and (15). Therefore, 7 GCPs at least are required to determine the RFCs.

$$
X=\frac{(1 p l l Z) \cdot\left(e_{0} e_{1} e_{2} e_{3}\right)^{T}}{(1 p l l Z) \cdot\left(1 g_{1} g_{2} g_{3}\right)^{T}}
$$




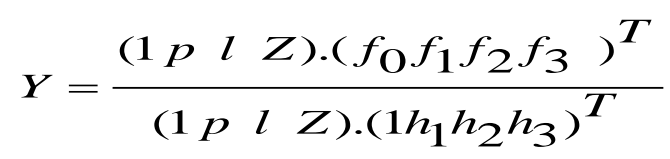

The equations from (10) to (15) utilizing four different polynomial $\left(F_{5}, F_{6}, F_{7}, F_{8}\right)$ with different orders to represent the first, second and third order of inverse RFMs. The order of the used polynomial is assign according to the applied RFM. Actually, these equations provide the comprehensive first, second and third order RFMs. However, some modification can be introduced to the general form of RFMs to simplify the solution and to reduce the number of unknowns (RFCs).

Applying the general inverse RFM but considering the two denominators of the two equations are equal to 1.0. In other words, F6 = F8 =1.0. In this case, the regular inverse polynomial model will be resulted and there is no longer a ration between two polynomial functions. Consequently, the generalized form of the 3D inverse polynomial model is as follows [9]:

$$
\begin{aligned}
& X=\left(\begin{array}{llll}
1 p & l & Z & \ldots l^{3} Z^{3}
\end{array}\right) \cdot\left(e_{0} e_{1} e_{2} e_{3} \ldots e_{18} e_{19}\right)^{T} \\
& Y=\left(\begin{array}{lllll}
1 & p & l & Z & \ldots l^{3} Z^{3}
\end{array}\right) \cdot\left(f_{0} f_{1} f_{2} f_{3} \ldots f_{18} f_{19}\right)^{T}
\end{aligned}
$$

There are 40 unknown coefficients in the third order 3D inverse polynomial and 20 polynomial coefficients in each equation of the third order polynomial model as shown in equations. At least 20 ground control points (GCPs) are required to solve the third order $3 \mathrm{D}$ inverse polynomial. The second order 3D inverse polynomial has 20 polynomial coefficients and 10 polynomial coefficients in each equation of the second order polynomial model as in equations (18) and (19). So, 10 GCPs at least are required to determine the unknown coefficients.

$$
\begin{aligned}
& X=\left(\begin{array}{lllll}
1 & p & l & Z & \ldots l^{2} Z^{2}
\end{array}\right) \cdot\left(e_{0} e_{1} e_{2} e_{3} \ldots e_{8} e_{9}\right)^{T} \\
& Y=\left(\begin{array}{lllll}
1 & p & l & Z & \ldots l^{2} Z^{2}
\end{array}\right) \cdot\left(f_{0} f_{1} f_{2} f_{3} \ldots f_{8} f_{9}\right)^{T}
\end{aligned}
$$

The first order inverse 3D inverse polynomial has 8 polynomial coefficients and 4 unknowns in each equation as shown in equations (20) and (21). In such a case, 4 GCPs at least are required to determine the polynomial coefficients [10].

$$
\begin{aligned}
& X=\left(\begin{array}{lll}
1 p & l & Z
\end{array}\right) \cdot\left(e_{\mathrm{O}} e_{1} e_{2} e_{3}\right)^{T} \\
& Y=\left(\begin{array}{lll}
1 p & l & Z
\end{array}\right) \cdot\left(f_{\mathrm{O}} f_{1} f_{2} f_{3}\right)^{T}
\end{aligned}
$$

\section{DATA SOURCES}

Tanta test area covers a part of a cultivated region of Tanta city, which is a part of Nile Delta in Egypt. Tanta is the capital of El-Gharbiya Governorate and it is situated 92 kilometers to the North of Cairo, the Egyptian capital. A subscene was cut out of a panchromatic GeoEye image acquired on May 11, 2011. The subscene size is 6000 pixel by 6000 pixel and the ground resolution is 0.50 meter. Fig. 1 shows the GeoEye subscene of Tanta study area.

A digital map of Tanta city produced by the Egyptian General Survey Authority (EGSA) of scale 1: 5000 was used as a base reference data. The vector map was extracted from this digital map with the aid of AutoCAD Map 3D software. In addition, the reference DTM was extracted from the digital map spot heights with the aid of Surfer 13 software.

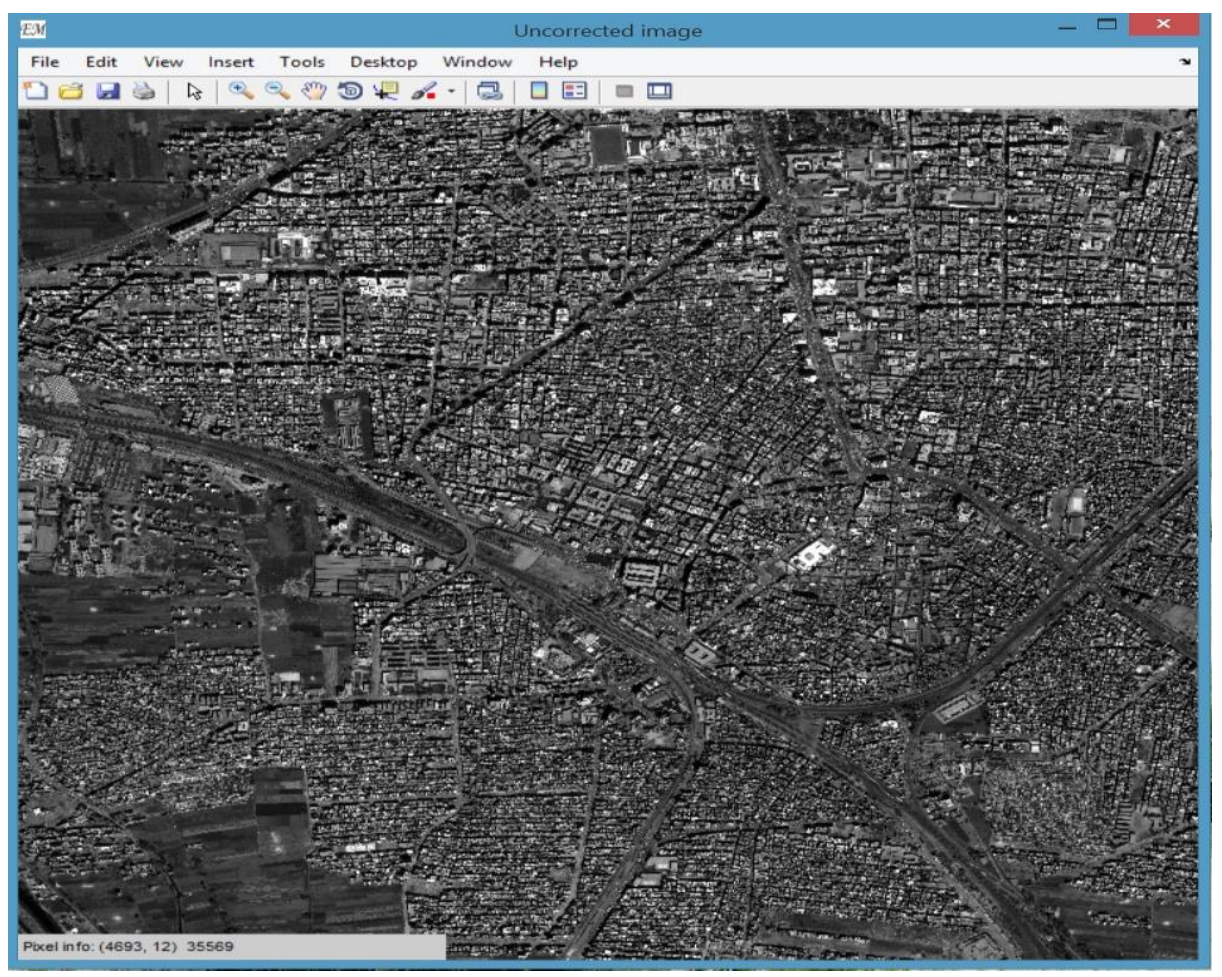

Fig. 1 GeoEye subscene of Tanta area 


\section{RESULTS AND ANALYSIS}

The three dimensional coordinates of 63 control points were collected from Tanta data set using control points selection tool of EMAN software. Out of these 63 control points, 49 control points were chosen as GCPs for the actual computation of the model coefficients and the remaining 14 control points were used as check points (CKPs). The GCPs were selected so that they are well distributed and spaced uniformly throughout the study area. The projection system of the coordinates is Universal Transverse Mercator (UTM) and the reference ellipsoid is WGS84.

For the third, second, first order of RFM and polynomial model different numbers of GCPs were used starting at 49 points, and then the number was reduced till the minimum required number for each order is reached.

The accuracy is expressed as the root mean square error (RMSE) of the residuals in X, and Y directions. The RMSE of CKPs in the inverse models can be derived by the following equations:

$$
\begin{aligned}
& \operatorname{RMSE}(X)=\sqrt{\frac{\sum_{i=1}^{n}\left(\Delta X_{i}\right)^{2}}{n-1}} \\
& \operatorname{RMSE}(Y)=\sqrt{\frac{\sum_{i=1}^{n}\left(\Delta Y_{i}\right)^{2}}{n-1}} \\
& \operatorname{RMSE}(T)=\sqrt{\frac{\sum_{i=1}^{n}\left(\left(\Delta X_{i}\right)^{2}+\left(\Delta Y_{i}\right)^{2}\right)}{n-1}}
\end{aligned}
$$

Where:

$$
\begin{array}{ll}
n & =\text { Number of check control points } \\
\Delta X, \Delta Y & =\text { Residual of ground coordinates of CKPs } \\
R M S E(X) & \begin{array}{l}
=\text { Root mean square error of CKPs in } \mathrm{X} \\
\text { direction }
\end{array} \\
R M S E(Y) & \begin{array}{l}
=\text { Root mean square error of CKPs in } \mathrm{Y} \\
\text { direction }
\end{array} \\
R M S E(T) & =\text { Total root mean square error of CKPs }
\end{array}
$$

Table I shows the RMSEs of ground coordinates for 14 CKPs resulted using the three RFM orders and different numbers of GCPs by PCI software. The corresponding RMSEs of CKPs resulted using EMAN software are presented in Table II.

By comparing the corresponding results from table I where PCI was used and table II where EMAN software was used, it is obvious that for different orders of RFMs the resulted RMSE of CKPs due to using EMAN software are very close and almost similar to their corresponding results obtained using PCI software. The maximum difference is less than $4 \%$ of RMSE of CKPs and in most experiments, the difference is less than $2 \%$ of the RMSE of CKPs. Thus, the capability and efficiency of EMAN software have been proved to geometrically correct the remotely sensed images using RFMs with different orders.

As PCI software does not support the 3D polynomial models, the results obtained by EMAN software using these models and using different number of GCPs were compared to their corresponding results obtained using EMAN software using RFMs. However, it was previously proved that EMAN software could provide very close planimetric accuracy as PCI software in case of using RFMs to geometrically correct the GeoEye images. Table III shows the RMSEs of CKPs due to applying the 3D polynomial models with different numbers of GCPs using EMAN the new standalone software.

However, it is clear from the results in tables (II and III) that the obtained EMSE from EMAN software due to using 3D polynomial models with different numbers of GCPs are stable, reasonable and comparable to the corresponding RMSEs obtained using the RFMs. Moreover, it is feasible to obtain an acceptable planimetric accuracy from 3D polynomial model using less number of GCPs rather than the number of GCPs required for 3D RFMs. For example, for the third order polynomial model with 30 GCPs the RMSE of CKPs is 0.53 meter while for the third order RFM with 45 GCPs the RMSE of CKPs is 0.55 meter.

Fig. 2 shows the graphical representation of relationship between the calculated RMSEs of CKPs and the used number of GCPs using different 3D RFM and 3D polynomial model. The 3D polynomial models of different orders have provided RMSE that is comparable and close to the RFMs as shown in fig. 2.

\section{CONCLUSION}

This study was carried out to investigate the performance of the new standalone EMAN software regarding the rectification of GeoEye image using different orders of 3D polynomial model and using different numbers of GCPs. unfortunately, the different orders of 3D polynomial model are not supported by PCI software. However, this group of empirical models has performed properly as they are examined and evaluated in EMAN software.

The results demonstrated the capability of $3 \mathrm{D}$ polynomial models for rectifying GeoEye images since it provides competitive geometric accuracies, compared to those obtained using 3D RFMs. In addition to the simplicity and computation speed of the 3D polynomial models, these models require considerably less number of GCPs compared to 3D RFMs. 
TABLE I. THE RMSE OF CKPS IN METERS RESULTED USING RFMS IN PCI SOFTWARE

\begin{tabular}{|c|c|c|c|c|c|c|c|c|c|}
\hline \multirow{2}{*}{$\begin{array}{c}\text { No. of } \\
\text { GCPs }\end{array}$} & \multicolumn{3}{|c|}{ Third order RFM } & \multicolumn{3}{c|}{ Second order RFM } & \multicolumn{3}{c|}{ First order RFM } \\
\hline & $\mathrm{X}$ & $\mathrm{Y}$ & $\mathrm{T}$ & $\mathrm{X}$ & $\mathrm{Y}$ & $\mathrm{T}$ & $\mathrm{X}$ & $\mathrm{Y}$ & $\mathrm{T}$ \\
\hline 49 & 0.33 & 0.34 & 0.47 & 0.38 & 0.36 & 0.52 & 0.38 & 0.34 & 0.51 \\
\hline 45 & 0.40 & 0.35 & 0.53 & 0.40 & 0.37 & 0.54 & 0.37 & 0.35 & 0.51 \\
\hline 40 & 0.43 & 0.41 & 0.59 & 0.40 & 0.42 & 0.58 & 0.36 & 0.35 & 0.50 \\
\hline 39 & 0.44 & 0.40 & 0.59 & 0.44 & 0.42 & 0.61 & 0.38 & 0.34 & 0.51 \\
\hline 35 & & & & 0.40 & 0.49 & 0.63 & 0.36 & 0.39 & 0.53 \\
\hline 30 & & & & 0.41 & 0.44 & 0.60 & 0.35 & 0.39 & 0.52 \\
\hline 25 & & & & 0.44 & 0.39 & 0.59 & 0.38 & 0.40 & 0.55 \\
\hline 20 & & & & 0.41 & 0.40 & 0.57 & 0.35 & 0.41 & 0.54 \\
\hline 19 & & & & 0.42 & 0.41 & 0.59 & 0.35 & 0.47 & 0.59 \\
\hline 15 & & & & & & & 0.39 & 0.47 & 0.61 \\
\hline 10 & & & & & & & 0.55 & 0.52 & 0.76 \\
\hline 7 & & & & & & & 0.66 & 0.93 & 1.14 \\
\hline
\end{tabular}

TABLE II. THE RMSE OF CKPS IN METERS RESULTED USING RFMS IN EMAN SOFTWARE

\begin{tabular}{|c|c|c|c|c|c|c|c|c|c|}
\hline \multirow{2}{*}{$\begin{array}{c}\text { No. of } \\
\text { GCPs }\end{array}$} & \multicolumn{3}{|c|}{ Third order RFM } & \multicolumn{3}{c|}{ Second order RFM } & \multicolumn{3}{c|}{ First order RFM } \\
\cline { 2 - 10 } & $\mathrm{X}$ & $\mathrm{Y}$ & $\mathrm{T}$ & $\mathrm{X}$ & $\mathrm{Y}$ & $\mathrm{T}$ & $\mathrm{X}$ & $\mathrm{Y}$ & $\mathrm{T}$ \\
\hline 49 & 0.33 & 0.33 & 0.47 & 0.38 & 0.36 & 0.52 & 0.38 & 0.34 & 0.51 \\
\hline 45 & 0.41 & 0.36 & 0.55 & 0.40 & 0.37 & 0.54 & 0.37 & 0.35 & 0.51 \\
\hline 40 & 0.45 & 0.41 & 0.61 & 0.40 & 0.41 & 0.58 & 0.36 & 0.35 & 0.50 \\
\hline 39 & 0.43 & 0.42 & 0.60 & 0.44 & 0.42 & 0.61 & 0.38 & 0.34 & 0.51 \\
\hline 35 & & & & 0.41 & 0.49 & 0.64 & 0.36 & 0.39 & 0.53 \\
\hline 30 & & & & 0.41 & 0.44 & 0.60 & 0.35 & 0.39 & 0.53 \\
\hline 25 & & & & 0.44 & 0.38 & 0.59 & 0.38 & 0.40 & 0.55 \\
\hline 20 & & & & 0.41 & 0.40 & 0.57 & 0.35 & 0.41 & 0.54 \\
\hline 19 & & & & 0.42 & 0.41 & 0.58 & 0.35 & 0.47 & 0.58 \\
\hline 15 & & & & & & & 0.39 & 0.47 & 0.61 \\
\hline 10 & & & & & & & 0.55 & 0.52 & 0.75 \\
\hline 7 & & & & & & & 0.65 & 0.94 & 1.14 \\
\hline
\end{tabular}

TABLE III. THE RMSE OF CKPS IN METERS RESULTED USING PMS IN EMAN SOFTWARE

\begin{tabular}{|c|c|c|c|c|c|c|c|c|c|}
\hline \multirow{2}{*}{$\begin{array}{c}\text { No. of } \\
\text { GCPs }\end{array}$} & \multicolumn{3}{|c|}{ Third order PM } & \multicolumn{3}{c|}{ Second order PM } & \multicolumn{3}{c|}{ First order PM } \\
\hline & $\mathrm{X}$ & $\mathrm{Y}$ & $\mathrm{T}$ & $\mathrm{X}$ & $\mathrm{Y}$ & $\mathrm{T}$ & $\mathrm{X}$ & $\mathrm{Y}$ & $\mathrm{T}$ \\
\hline 49 & 0.35 & 0.35 & 0.49 & 0.42 & 0.35 & 0.55 & 0.40 & 0.37 & 0.54 \\
\hline 45 & 0.35 & 0.35 & 0.50 & 0.42 & 0.36 & 0.56 & 0.39 & 0.37 & 0.54 \\
\hline 40 & 0.37 & 0.43 & 0.56 & 0.41 & 0.36 & 0.55 & 0.40 & 0.37 & 0.54 \\
\hline 39 & 0.38 & 0.47 & 0.61 & 0.40 & 0.34 & 0.54 & 0.41 & 0.36 & 0.55 \\
\hline 35 & 0.36 & 0.45 & 0.58 & 0.42 & 0.39 & 0.57 & 0.40 & 0.38 & 0.55 \\
\hline 30 & 0.39 & 0.36 & 0.53 & 0.42 & 0.38 & 0.57 & 0.40 & 0.39 & 0.55 \\
\hline 25 & 0.66 & 0.64 & 0.92 & 0.44 & 0.37 & 0.58 & 0.39 & 0.38 & 0.55 \\
\hline 20 & 1.21 & 2.74 & 3.00 & 0.45 & 0.38 & 0.59 & 0.40 & 0.38 & 0.55 \\
\hline 19 & & & & 0.44 & 0.40 & 0.60 & 0.42 & 0.38 & 0.57 \\
\hline 15 & & & & 0.46 & 0.48 & 0.66 & 0.47 & 0.39 & 0.61 \\
\hline 10 & & & & 0.88 & 1.23 & 1.51 & 0.46 & 0.42 & 0.63 \\
\hline 7 & & & & & & & 0.56 & 0.46 & 0.72 \\
\hline 5 & & & & & & & 0.58 & 0.54 & 0.79 \\
\hline 4 & & & & & & & 1.27 & 0.98 & 1.60 \\
\hline
\end{tabular}



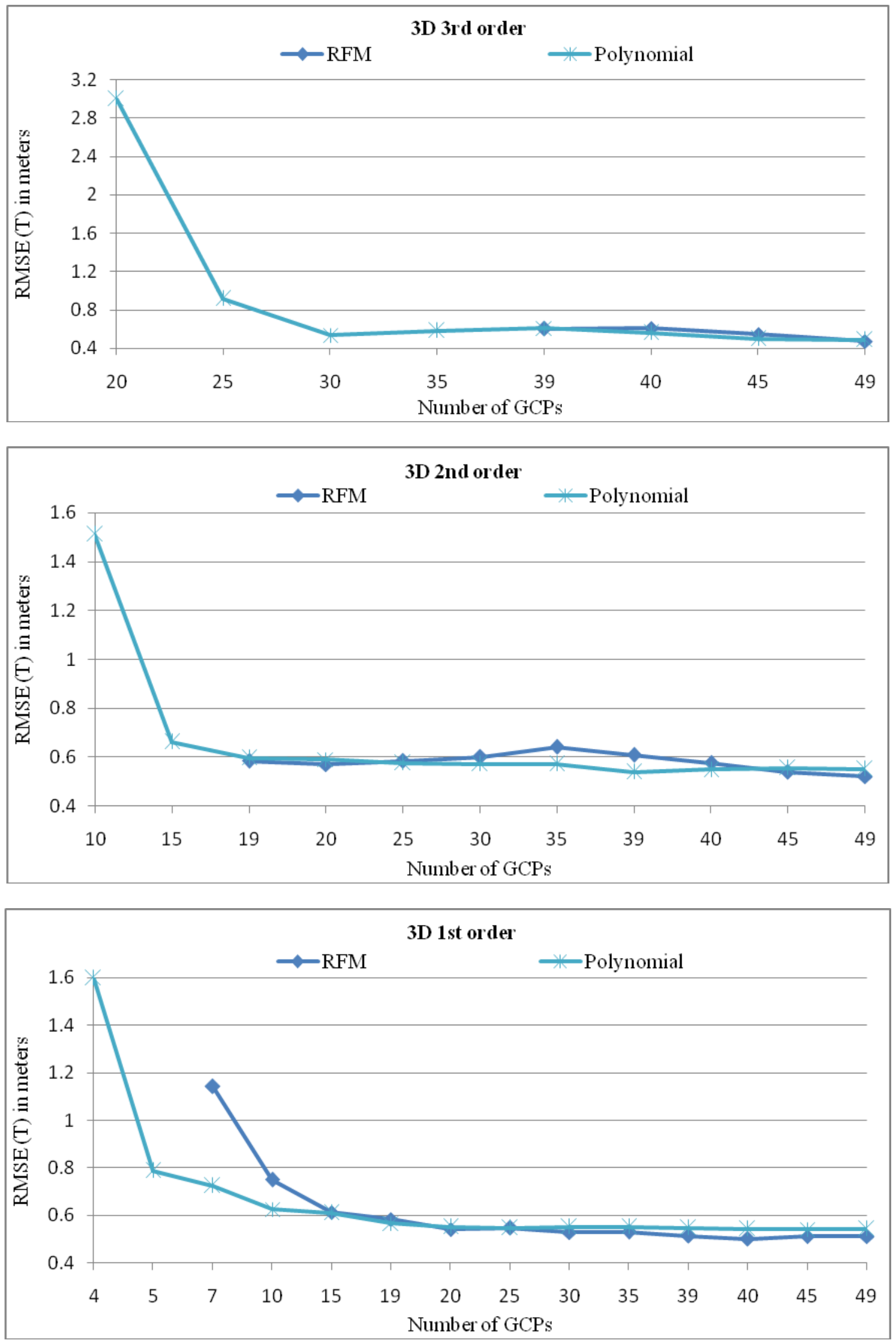

Fig. 2 The RMSE of CKPs for 3D models at different numbers of GCPs 


\section{REFERENCES}

[1] Toutin, T., "Review article: geometric processing of remote sensing images: models, algorithms and methods". International Journal of Remote Sensing Vol.25, No.10, 1893-1924, 2004.

[2] DigitalGlobe, GeoEye -1 datasheet, 2013.

[3] Maglione, P., Parente, C., Vallario, A., 2013 "Using Rational Polynomial Functions for rectification of GeoEye-1 imagery" IOSR Journal of Applied Geology and Geophysics Vol.1, No.6, 11-17.

[4] Fraser, C. S. \& Ravanbakhsh, M., 2009. "Georeferencing from Geoeye-1 Imagery: Early Indications of Metric Performance". Paper presented at ISPRS Hannover Workshop, Hannover. International Society for photogrammetry and Remote Sensing.

[5] Tao, V., Hu, Y., 2002. "3D reconstruction methods based on the rational function model". Photogrammetric Engineering \& Remote Sensing, Vol. 68 (7), 705-714.

[6] OGC, 1999, The Open GISTM Abstract Specifications: The Earth Imagery Case, Vol. 7,http://www.opengis.org/techno/specs/htm/.

[7] Tao, C.V., Hu, Y., 2001a. "A comprehensive study on the rational function model for photogrammetric processing", Photogrammetry Engineering and Remote Sensing, Vol.67, No. 12, pp. 1347-1357

[8] Afify, H. and Zhang, Y., 2008. "Accuracy Assessment of User-Derived RFCs for Ortho-Rectification of HighResolution Satellite Imagery", International Journal of Geoinformatics, Vol. 4, No. 4, 17-24.

[9] Tao, V., Hu, Y., 2001. "Use of the rational function model for image rectification", Canadian Journal of Remote Sensing, Vol. 27 , No. 6, 593-602.

[10] Yamakawa, T. and Fraser, C.S., 2004. "The affine projection model for sensor orientation: experiences with high-resolution satellite imagery". XX. Congress of International Society for Photogrammetry and Remote Sensing (ISPRS), Commission I, Working Group IV/5, 12-23 July 2004, İstanbul-Türkiye. 